\title{
Coupled Compression RTM and Composite Layup Optimization
}

\author{
R. Le Riche ${ }^{1}$, A. Saouab ${ }^{2}$ and J. Bréard ${ }^{2}$ \\ ${ }^{1}$ CNRS URA 1884 / SMS, Ecole des Mines de Saint Etienne, \\ France, leriche@emse.fr (corresponding author). \\ ${ }^{2}$ Lab. de Mécanique, Univ. du Havre, France.
}

With additional erratum on $(S O)$ formulation as compared to the published version in Composites Science and Technology, Vol.63, No.15, Elsevier publ., July 2003, pp. 2277-2287.

\begin{abstract}
A methodology for studying the implications of taking into account manufacturing at early design stages is presented. It is applied to a rectangular laminate made by RTM and compression (RTCM). The plate is designed for injection time, maximum mold pressure, stiffness and buckling. Semi-analytical, numerically inexpensive models of the processes and structure enable a thorough investigation of the couplings between process and structure by comparing four design formulations: in decoupled problems, either the process or the structure is optimized; then, the process is optimized with targetted low or high structural performance. A globalized Nelder-Mead optimization algorithm is used. The rigour of the method and the relative simplicity of the application case provide a clear description of how maximum mold pressures, injection times and final structures properties are tradedoff.
\end{abstract}

Keywords : Optimization, Resin transfer moulding (E), Laminates (C), Porosity (B), Buckling (C).

\section{Introduction}

After having been praised for their mechanical properties, composite materials have lately received much attention with regard to reducing their manufacturing cost. The possibility of automating composite structure production by processes based on resin transfer molding or resin infusion is seen as an important factor for controlling costs $([1])$. 
Choosing a structural design (which includes materials) and a manufacturing process are coupled decisions ([2]). While early composite design studies simplified the analysis by separating structural design ([3]) and process tuning ([4]), in the last decade cost has increasingly been considered at an early stage.

A series of works focus on tuning a particular process, e.g., Resin Transfer Molding (RTM), and sizing the structure simultaneously $([5,6])$.

Other contributions estimate cost in terms of structural complexity, independent of the process. In [7, 8], ease of prepreg layup is considered as one of the design criteria for general structures along with strength. In [9], the complexity depends on the geometry of ribs and spars of a composite box.

Finally, in [10] and [11, 12], different manufacturing routes are compared using cost models.

In terms of optimization methods, most of the aforementioned works rely on enumeration $([10,5])$ or problem decomposition and local searches ([7, 12]). Some attempts have been made at using more robust, but numerically more expensive, global optimization algorithms (e.g., genetic algorithms in $[4,6])$. Also, because many criteria need to be traded off against one another, multi-criteria approaches have appeared in [12, 9].

This article presents a methodology to study the effects of taking into consideration the process at early design stages in a numerically well-founded fashion. A simple, yet common, scenario is considered: a rectangular plate is manufactured by resin transfer molding and compression (RTCM). Injection can be flow rate or pressure controlled and compression can follow or be simultaneous with injection, which means that there are four processes to study. Process criteria are maximum mold pressure and injection time. They affect tooling quality and time to market. They are therefore partly representative of manufacturing cost. Structural criteria are stiffness and buckling. Under these assumptions, semi-analytical models of the process and structure are available. These models are numerically inexpensive, which enables the use of a global optimization algorithm. The process parameters and the ply orientations are the design variables. The effects of required structural performance and

maximum

allowable mold pressure on optimal processes and designs are characterized by solving coupled optimization problems. 


\section{Design formulation}

\subsection{Structural and RTCM couplings in composites}

When considering the RTCM process, engineers are typically concerned with tuning parameters such as injection pressure/flow rate, temperature, gate/vents positions and preform characteristics. The criteria they observe are the injection time, the maximum mold pressure, the temperature distribution, preform damage, void contents and degree of curing. At the same time, structural designers specify materials, geometry, fiber volumes, fiber orientations and ply thicknesses to achieve minimum mass and specified strength, stiffness, damage or other failure criteria. An overview of process and structure variables and criteria involved in composites engineering is given in Fig. 1. While process and structure engineering are traditionally separate activities, the central part of the Figure shows variables and criteria that are common to both fields: preform characteristics, void content, polymerization and preform damage couple manufacturing and the final structure.

A method will shortly be introduced that estimates the consequences of accounting for these couplings in composite laminates. A channel flow model of the RTCM process ([13]) is used. Among all the RTCM processes that are described in Appendix A, four are compared here :

RTCM-P A successive two step process, starting with a pressure imposed injection, at $P$ constant, followed by compression at a constant rate, $R_{C} . I_{P} \%$ of the laminate length is filled only by injection before compression starts, i.e., compression may be applied before the laminate is fully infiltrated.

RTCM-F Same as above, except that injection is performed at a constant flow rate $R_{F}$.

RTCM-SP Pressure controlled injection and compression are simultaneous.

RTCM-SF Flow rate controlled injection and compression are simultaneous.

Note that these process models assume a closed mold, i.e., no resin can be vented in the compression phase. In this case, when $I_{P}=100 \%$ or $R_{C}=0 \mathrm{~m} / \mathrm{s}$, the RTCM process degenerates into a RTM process. 


\subsection{Accounting for process and structure in the opti- mization problem formulation}

The process-structure couplings first take place in the physical model of the system that is being optimized. They need to be taken into account further in the optimization problem formulation. This is achieved when choosing design variables and criteria.

There are two possible formulations for a coupled design problem. One can solve a multi-objective optimization problem (e.g., [12, 9]),

$$
\left\{\begin{array}{l}
\min _{x \in S} f_{1}(x) \\
\text { and } \min _{x \in S} f_{2}(x), \\
\cdots \\
\text { and } \min _{x \in S} f_{m+1}(x)
\end{array}\right.
$$

where $f_{i}$ are the criteria, $x$ is a vector of design variables and $S$ is the design space. Alternatively, a constrained single objective problem is formulated by transforming some criteria into inequalities, $g_{i}$,

$$
\left\{\begin{array}{l}
\min _{x \in S} f(x) \\
\text { and } g_{1}(x) \leq 0 \\
\cdots \\
\text { and } g_{m}(x) \leq 0
\end{array}\right.
$$

With respect to a criterion, a constraint requires an additional allowable value. As an example, the criterion associated with the maximum pressure during injection, $P_{\max }$, is

$$
\min P_{\max }
$$

while the constraint is

$$
\text { satisfy } P_{\max } \leq P_{\max }^{\text {allow }}
$$

The multi-objective approach, which does not need a priori allowable values, is the most general formulation. It has many - eventually, an infinite number of - solutions, called the Pareto set, which is all the possible compromises that can be struck between the criteria. However, from a numerical point of view, solving the multi-objective problem (1) is much more complex than solving the constrained single objective problem (2). State-of-the-art multiobjective algorithms are evolutionary algorithms (see [14] for a review) which 
have a slower convergence than other single objective algorithms such as the Globalized and Bounded Nelder-Mead algorithm (see Section 2.4 and [15]). Furthermore, the set of solutions need to be post-processed in order to decide which compromise should be manufactured.

In the current work, a complete multi-objective optimization is replaced with a series of four constrained single objective problems: a process only problem, $(P O)$, a structure only problem, $(S O)$, a coupled problem with low performing structure, $(C L)$, and a coupled problem with optimal structure (solution of the structure only problem), $(C O)$. Because the series is composed of constrained single objective problems, numerical resolution is eased. Extreme regions of the Pareto set are located in the process and structure only problems. The solution of the structure only problem is an indication of which allowable values can be achieved in the coupled problems. From a didactic point of view, comparing the solutions of the four problems shows how simultaneous consideration of the process and the structure affects the final design and its manufacturing.

\subsection{Detailed design problems formulations}

The series of four optimizations are repeated for the four processes RTCM-P, RTCM-F, RTCM-SP and RTCM-SF. In all processes, imposed pressures, flow rates, and compression rates are time constants (but optimization variables).

Although the proposed method can be applied to any laminate, symmetric and balanced laminates of eight layers are considered, so there are two design variables to describe the stacking sequence, $\theta_{1}$ and $\theta_{2}$. The $\theta^{\prime}$ 's are zero when the fibers are aligned with the resin flow. $I$ denotes the injection imposed condition, that is, either a pressure $P$ or a flow rate $R_{F}$. All design variables are continuous.

There are five design variables for processes that have successive injection and compression,

$$
x=\left(\left\{\begin{array}{c}
P \\
R_{F}
\end{array}, R_{C}, I_{P}, \theta_{1}, \theta_{2}\right),\right.
$$

and four design variables when injection and compression are simultaneous since $I_{P}$ has no meaning in this case,

$$
x=\left(\left\{\begin{array}{c}
P \\
R_{F}
\end{array}, R_{C}, \theta_{1}, \theta_{2}\right) .\right.
$$


The two process design criteria are the total injection time, $T_{P}$, and the maximum mold pressure, $P_{\max }$. Both measures are representative of the manufacturing cost. $T_{P}$ is a small part of the total production time which is dominated by the curing time. In practice however, the injection time is the main degree of freedom of the production time since the resin choice is limited. $P_{\max }$ is an indication of the tooling price. High $P_{\max }$ implies heavy metal molds while a low $P_{\max }$ suggests that a composite mold (like in RTMlight, cf. [16]) may be sufficient. Note that when injection and compression are successive, $T_{P}$ is the total time of the two phases. The models used to calculate $T_{P}$ and $P_{\max }$ are presented in Appendix A. In the RTCM-SP process, all combinations of pressure and compression rate are not valid. The pressure in the mold may become larger than the injection pressure, which causes an inversion of the resin flow at time $T_{\mathrm{inv}}$. This scenario is avoided by defining an inversion time constraint

$$
T_{P} \leq T_{\mathrm{inv}}
$$

which is added to all optimization problems involving the RTCM-SP process. Structural design criteria concern the transverse effective stiffness $E_{y}$, the shear effective stiffness $G_{x y}$ and the buckling load factor $\lambda$. Calculations of the structural criteria are based on the Classical Lamination Theory and are outlined in Appendix B. $E_{x}$, the longitudinal effective stiffness, may also be an important structural property. Yet, $E_{x}$ doesn't need to be included here because it is redundant with the process criteria, $T_{P}$ and $P_{\max }: E_{x}$, like $T_{P}$ and $P_{\max }$, drives the fiber orientation to $0^{\circ}$.

The process only problem, $(P O)$, is

$$
(P O) \quad\left\{\begin{array}{l}
\min _{I, R_{C}, I_{P}, \theta_{1}, \theta_{2}} T_{P} \\
\text { such that } P_{\max } \leq P_{\max }^{\text {allow }}
\end{array}\right.
$$

where $P_{\max }^{\text {allow }}$ is a user-defined allowed maximum mold pressure. More generally, allowable values are denoted with the allow superscript. The structure only problem, $(S O)$, is

$$
(S O) \quad\left\{\begin{array}{l}
\max _{I, R_{C}, I_{P}, \theta_{1}, \theta_{2}} E_{y} \\
\text { such that } G_{x y} \geq G_{x y}^{*} \\
\text { and } \lambda \geq \lambda^{*}
\end{array}\right.
$$

Let $E_{y}^{*}, G_{x y}^{*}$ and $\lambda^{*}$ denote the structural properties at a solution of the $(S O)$ problem. A first coupled process-structure problem, where the structure has 
a low quality, is

$$
(C L) \quad\left\{\begin{array}{l}
\min _{I, R_{C}, I_{P}, \theta_{1}, \theta_{2}} T_{P} \\
\text { such that } P_{\max } \leq P_{\max }^{\text {allow }} \\
E_{y} \geq E_{y}^{\text {allow }}, \\
G_{x y} \geq G_{x y}^{\text {allow }} \text { and } \\
\lambda \geq 1
\end{array}\right.
$$

This problem has a low structural quality because the allowable values in

the structural constraints are chosen such that $E_{y}^{\text {allow }}<E_{y}^{*}, G_{x y}^{\text {allow }}<G_{x y}^{*}$ and $\lambda^{\text {allow }}<\lambda^{*}$. Finally, the coupled process-structure problem with an optimal structure, $(C O)$, has the same expression as $(C L)$, but the allowable values of the structural criteria correspond to the solution of the structure only problem, i.e., $E_{y}^{\text {allow }}=E_{y}^{*}, G_{x y}^{\text {allow }}=G_{x y}^{*}$ and $\lambda^{\text {allow }}=\lambda^{*}$.

\subsection{The Globalized and Bounded Nelder-Mead opti- mization algorithm}

The constrained single objective optimization problems are dealt with by the Globalized and Bounded Nelder-Mead algorithm ([15]), GBNM. This algorithm is based on repeated direct Nelder-Mead (sometimes also called simplex, [17]) searches, which work on continuous variables, do not need gradient information and, individually, converge faster than probabilistic global search methods such as evolutionary algorithms ([18]). The method is made global by restarting local searches based on a probability density which biases new local searches towards unexplored regions of the design space $S$. Restarts are stopped when a user specified maximum number of analyses, $N_{\max }$, has been reached. If the maximum number of analyses is large enough and the problem has several local optima, the GBNM algorithm typically finds many of them. Note that degeneracy cases of the Nelder-Mead methods are detected and induce re-initialization of the simplex. Additionally, the GBNM algorithm copes with bounds on the variables by projection and general non-linear constraints by an adaptive linear penalty scheme.

\section{$3 \quad$ Numerical applications}

A plate of length $a=0.5 \mathrm{~m}$ and width $b=0.1 \mathrm{~m}$ subject to a bi-axial in-plane load, $N_{x}=200000 N, N_{y}=20000 N$, is considered. Its fiber and 
resin properties are : $E_{1, f}=80.10^{9} \mathrm{~Pa}, E_{2, f}=80.10^{9} \mathrm{~Pa}, \nu_{12, f}=0.22$, $G_{12, f}=35.210^{9} \mathrm{~Pa}, E_{m}=3.4510^{9} \mathrm{~Pa}, \nu_{m}=0.3$. The initial ply thickness (before compression) is $h_{i}(0)=1 \mathrm{~mm}$ with a fiber volume fraction $v_{f, i}(0)=0.4, i=1,8$. The resin viscosity is $\mu=0.16$ Pa.s. $\mu$ is assumed here to be a time constant, which is valid if the curing time is larger than the injection time. The coefficients of the permeability relations (cf. Equation (18)) are $A_{1}=1.710^{-12} \mathrm{~m}^{2}, B_{1}=-4.12, A_{2}=6.810^{-14} \mathrm{~m}^{2}$ and $B_{2}=-5.64$.

\subsection{Parametric study}

Before turning to a complete optimization of the system, a parametric study is performed that gives an insight into the design variables and criteria relationship. To simplify the interpretation, the set of optimization variables of Equation (5) or (6) is replaced with two variables, that are varied in turn: the fiber orientation $\theta$ of a $\left(( \pm \theta)_{2}\right)_{s}$ laminate, and the injection length before compression starts.

For comparison purposes in the parametric study, the imposed pressure and flow rates are chosen equivalent on average, i.e., they satisfy

$$
R_{F}=\frac{1}{2 \mu} H(0) P K_{x},
$$

where $K_{x}$ is the average longitudinal permeability (cf. Appendix A.3) and $H(0)$ is the initial total thickness. The process where injection is pressure controlled and simultaneous with compression is not studied here because under the above conditions and a compression rate $R_{C}=1.10^{-6} \mathrm{~m} / \mathrm{s}$, inversion will always occur.

In Figure 2, the variation of the design criteria as a function of the fiber orientation $\theta$ is plotted. The following observations can be done:

- The injection time (top-left plot) is independent of the fiber orientation when the flow rate is imposed, while it increases when pressure is imposed and the fibers are disoriented with respect to the injection direction (the permeability decreases).

- The maximum pressure during manufacturing (top-right plot) increases when the fibers are disoriented. The augmentation is drastic when injection flow rate is imposed and compression is simultaneous. The 
rise is much higher when flow rate is imposed than when pressure is imposed.

- The simultaneous process, with the given settings, yields a structure that is thicker than that of successive processes. In other words, the final fiber volume fraction, $V_{f}\left(T_{P}\right)$, is lower. This explains why in-plane stiffnesses are lower in the simultaneous process while the buckling load factor is larger. Indeed, the in-plane stiffnesses increase linearly with $V_{f} \equiv\left(1 /\right.$ thickness) while the buckling load increases with (thickness $\left.{ }^{3}\right)$. In terms of buckling, it is more efficient to sacrifice fiber fraction for a better flexural strength. Note that this observation is case dependent: for a sufficiently higher compression rate or a lower $I_{P}$ or a lower $R_{F}$, the simultaneous process yields thinner laminates than the successive processes do.

- It is confirmed that the optimal fiber orientation is $90^{\circ}$ for $E_{y}$ and $45^{\circ}$ for $G_{x y}$ and buckling.

- The successive processes, with the flow rate or the pressure imposed, produce structures with the same final thicknesses, therefore the same structural criteria. Indeed, the final thickness depends only on the compression phase, which is the same in both cases $\left(R_{C}\right.$ and $I_{P}$ are identical).

Design criteria are represented as a function of the percentage of plate length filled by injection before compression starts, $I_{P}$, in Figure 3 under the same material and process conditions as in Figure 2. The following simple comments can be made:

- The compression rate is slow with respect to the injection flow rate or pressure, so the injection time decreases with $I_{P}$. If the compression rate were higher, opposite conclusions would be drawn.

- For successive processes, the maximum pressure decreases with $I_{P}$ as long as it occurs during compression. Reciprocally, when maximum pressure occurs during injection, it increases with $I_{P}$. The top right plot of Figure 3, for RTCM-F and $I_{P}$ beyond $45 \%$ gives such an example.

The results of the parametric study are specific to the material chosen and process tuning. No monotony in the criteria variation as a function of the 
variables exists. In order to properly account for all parameter combinations, it is necessary to optimize the system with a global non-linear algorithm.

\section{$3.2 \quad$ Optimization results}

The GBNM algorithm (see paragraph 2.4) is applied to 32 optimization problems. Each search is $N_{\max }=80000$ analyses long. Depending on the process, the average $\mathrm{CPU}$ time for one analysis ranges from 0.02 to $0.2 \mathrm{~s}$ on a $800 \mathrm{M} \mathrm{Hz}$ I686 Intel ${ }^{\mathrm{TM}}$ Pentium processor. The 32 problems correspond to the 4 formulations, process only $(P O)$, structure only $(S O)$, coupled low structural performance $(C L)$ and coupled optimal structure $(C O)$, repeated with the four processes (RTCM-P, RTCM-F, RTCM-SP, RTCM-SF) and two maximum pressures in the mold, $P_{\max }^{\text {allow }}=10.10^{5}$ and $6.10^{5} \mathrm{~Pa}$, respectively. The stacking sequence considered is $\left( \pm \theta_{1} / \pm \theta_{2}\right)_{s}$. The design variables are bounded as follows: $0.510^{5} \leq P \leq 6.10^{5} \mathrm{~Pa}, 0.510^{-7} \leq R_{F} \leq 100.10^{-7} \mathrm{~m}^{3} / \mathrm{s}$, $0 . \leq I_{P} \leq 100 . \%, 0 . \leq R_{C} \leq 0.410^{-3} \mathrm{~m} / \mathrm{s}, 0 . \leq \theta \leq 90{ }^{\circ}$. Allowable criteria values are $E_{y}^{\text {allow }}=15.10^{9} \mathrm{~Pa}, G_{x y}^{\text {allow }}=5.10^{9} \mathrm{~Pa}$ and $\lambda^{\text {allow }}=1$. in $(C L)$. Optimal values are $G_{x y}=6.89 \mathrm{GPa}$ and $\lambda^{*}=1.3$ in $(S O)$. By definition, mechanical allowable values in $(C O)$ stem from the solution of the $(S O)$ problem (see Paragraph 2.3). The results of the optimizations are given in Tables 1 to 5 .

The principal significance of these results consist in the numerical solutions to the coupled process-structure problems, $(C L)$ and $(C O)$.

$(C L)$ problem : at $P_{\max }^{\text {allow }}=10.10^{5} \mathrm{~Pa}$, RTCM-P is optimal with an injection time of $326 \mathrm{~s}$. Active constraints are $P_{\max }$ and $E_{y}$. RTCM-F and RTCM-SP are second best processes with a time of $390 \mathrm{~s}$. It should be noted that RTCM-SP is a gentle process because it results in a lower mold pressure $\left(6.10^{5} \mathrm{~Pa}\right.$ maximum). If $P_{\max }^{\text {allow }}$ is decreased to $6.10^{5} \mathrm{~Pa}$, a pressure controlled injection without compression is optimal in $391 \mathrm{~s}$. For low mold pressure, RTM is the optimal process, and both RTCM-P and RTCM-SP can degenerate into it.

$(C O)$ problem : RTCM-P is again the optimal process for both $P_{\max }^{\text {allow }}=10.10^{5}$ and $6.10^{5} \mathrm{~Pa}$ with injection times of 785 and $912 \mathrm{~s}$, respectively. All constraints are active (structural constraints are active by construction of the $(C O)$ problem).

Comparing $(C L)$ with $(C O)$ results shows that high structural performance more than doubles the injection time. In other words, when aiming 
at low structural performance, an uncoupled design procedure where, first, the layup is optimized and, second, the process is tuned, doubles the injection time with respect to a coupled procedure, $(C L)$.

Solutions of the process only and structure only problems are familiar to many experts. Yet, in order to be comprehensive, they are now commented. Secondarily, these classical solutions validate the numerical optimizers implemented.

Process only problem : fibers are oriented along the resin flow, $\theta_{1}=$ $\theta_{2}=0$. Compression is useful to successive processes, except RTCM$\mathrm{P}$ at low $P_{\max }^{\text {allow }}$. Compression never speeds up simultaneous processes because of the associated loss of permeability.

Structure only : loss of solution uniqueness for all processes, the structure only problems $(S O)$ have an infinite number of solutions, corresponding to all injection and compression settings that produce the same final thickness. These solutions are not equivalent in terms of injection time or mold pressure, but these criteria are not considered in $(S O)$. It can be seen for the RTCM-P process in Table 1 on the (SO) line. For RTCM-P and a fixed $I_{P}$, any combination of $P$ and $R_{C}$ results in the same final thickness, which is a resin conservation property. Indeed, $I_{P}$ sets the amount of resin injected in the mold which subsequently determines how much compression is needed to fill the plate.

Finally, when interpreting the optimization results in terms of process features, the following well-known traits underlie the solutions.

Compression : compression improves in-plane structural stiffnesses and reduces injection times at the expense of larger mold pressures. Therefore, when high structural performance is not required and mold pressure should remain low $\left((C L)\right.$ problem, $\left.P_{\max }^{\text {allow }}=6.10^{5} \mathrm{~Pa}\right)$, all optimal processes but RTCM-F have no compression. In the simultaneous processes, since compression induces fast mold pressure growth (it reduces permeability), it is absent in all simultaneous processes for low structural quality. In the $(C O)$ formulations, compression is necessary to achieve high structural performance.

Flow control : flow controlled injection is not optimal in our numerical applications because, for an equivalent injection time, it yields higher 
mold pressure than pressure controlled injection. RTCM-F optimal settings all use compression in a way such that $P_{\max }^{\text {allow }}$ is reached during both injection and compression steps. Nevertheless, flow controlled processes regain competitiveness for shorter plates.

Simultaneous processes : RTCM-SP and RTCM-SF are not optimal for the problems considered here. However, for low mold pressures and high structural properties, RTCM-SP has a strong potential. When

$P_{\max }^{\text {allow }}$ drops from $10.10^{5} \mathrm{~Pa}$ to $6.10^{5} \mathrm{~Pa}$, its time excess with respect to the optimal process shrinks from to 20 to $9 \%$.

\section{Concluding remarks}

The effects of accounting, at the first design stages, for the couplings between process and structure have been investigated in the case of an injected and compressed laminated plate.

The study is based on four design formulations, two where the couplings are ignored and either the structure or the process are optimized, and two coupled formulations aiming at obtaining low and high structural performances, respectively. Four processes, which have pressure or flow controlled injection and simultaneous or successive injection and compression phases are compared. Solutions analyses is based on semi-analytical process and structure models, whose numerical efficiency authorizes a global optimization by the GBNM algorithm.

The optimizations exhibit the fundamental trade-offs that exist between injection time, maximum mold pressure, structural in-plane and flexural stiffnesses. For example, the manufacturing of a low performance laminate by the optimal RTCM-P process needs an extra minute when the maximum pressure decreases from 10 to $6.10^{5} \mathrm{~Pa}$. If a high structural performance is targetted, the injection time increases by two minutes.

In terms of perspectives, this work lays the foundations for an approach to select, based on a final structure, a resin molding techniques from a wider panel of processes (Advanced RTM, Liquid Resin Infusion, Resin Film Infusion, ....). 


\section{References}

[1] Hinrichsen, J and Bautista C. 2001: The Challenge of Reducing both Airframe Weight and Manufacturing Cost. Air \& Space Europe, 3(3/4), $119-121$

[2] Ashby, M.F., Bréchet, Y.J.M., Cebon, D. and Salvo, L. 2003: Selection Strategies for Materials and Processes. to be published in J. of Modelling and Computer Simulations in Materials Sc. and Engng.

[3] Schmit, L.A. and Farshi, B. 1973: Optimum Laminate Design for Strength and Stiffness. Int. J. of Num. Meth. Engng., 7, 519-536

[4] Young, W.-B. 1994: Gate Location Optimization in Liquid Composite Moulding using Genetic Algorithms. J. Compos. Mater., 28(12), 10981113

[5] Nardari, C., Ferret, B. and Gay D. 2002: Simultaneous Engineering in Design and Manufacture using the RTM Process. Composites: Part A, 33, 191-196

[6] Park, C.H., Lee, W.I., Han, W.S. and Vautrin A. 2002: Weight minimization of composite laminated plates with multiple constraints. submitted for publication in Composites Science and Technology, November 2002

[7] Fine, A.S. and Springer G.S. 1997: Design of Composite Laminates for Strength, Weight, and Manufacturability. J. of Composite Materials, 31(23), 2330-2390

[8] Manne, P.M. and Tsai S.W. 1998: Design Optimization of Composite Plates : Part 1 -Design Criteria for Strength, Stiffness, and Manufacturing Complexity of Composite Laminates. J. of Composite Materials, 32(6), 544-571

[9] Wang, K., Kelly, D. and Sutton S. 2002: Multi-objective Optimisation of Composite Aerospace Structures. Composite Structures, 57, 141-148

[10] Bader, M.G. 2002: Selection of Composite Materials and Manufacturing Routes for Cost-effective Performance. Composites: Part A, 33, 913-934 
[11] Kassapoglou, C. 1999: Minimum Cost and Weight Design of Fuselage Frames - Part A : Design Constraints and Manufacturing Process Characteristics, Composites Part A, 30, 887-894

[12] Kassapoglou, C. 1999: Minimum Cost and Weight Design of Fuselage Frames - Part B : Cost Considerations, Optimization and Results, Composites:Part A, 30, 895-904

[13] Saouab, A., Bréard, J., and Bouquet, G. 2002: Contribution to the optimization of RTM and CRTM processes. Proc. of the 5th Intl. ESAFORM Conf. on Material Forming, 299-302

[14] Zitzler, E., and Thiele, L. 1998: Multiobjective Optimization Using Evolutionary Algorithms - A Comparative Case Study. Proc. of Parallel Problem Solving from Nature - PPSN V, Springer, Amsterdam, The Netherlands, September 1998, pp. 292-301.

[15] Luersen, M.A. and Le Riche, R. 2002: Globalized Nelder-Mead Method for Engineering Optimization. Proc. of the Third International Conference on Engineering Computational Technology, Paper 65, Civil-Comp Press, Prague, Czech Republic, 4-6 September 2002.

[16] Williams, C., Summerscales, J. and Grove S. 1996: Resin Infusion under Flexible Tooling (RIFT): a review. Composites Part A: Applied Science and Manufacturing, textbf27(7), 517-524

[17] Nelder, J.A., and Mead, R. 1965: A Simplex for Function Minimization. Computer J., 7, 308-313

[18] Bäck, T. 1996: Evolutionary Algorithms in Theory and Practice. Oxford: Oxford Univ. Press

[19] Pham, X.T., Trochu, F. and Gauvin, R. 1998: Simulation of Compression Resin Transfer Molding with Displacement Control. J. of Reinforced Plastics and Composites, 17, 1525-1556

[20] Chamis, C. C. 1984: Simplified Composite Micromechanics Equations for Hygral, Thermal, and Mechanical Properties. Sampe Quaterly, April 1984, 14-23. 
[21] Berthelot, J.-M. 1999: Composite Materials: Mechanical Behavior and Structural Analysis. Mechanical Engineering Series, Springer.

[22] Haftka, R.T., and Gürdal, Z. 1992: Elements of Structural Optimization. 3rd rev. and expanded ed., Kluwer Academic Publishers 


\section{A RTCM process analysis}

\section{A.1 A family of RTCM processes}

The generic resin transfer and compression molding scenario considered in this study is the case of a rectangular plate injected on one side and uniformly compressed (cf. Figure 4). If one further assumes a channel-like flow, the analysis is one-dimensional.

Many combinations of the RTCM process settings are possible. They make up a family of processes, of which four are optimized in the body of this article : the injection is either pressure or flow rate controlled at constant $P$ or $R_{F}$ respectively. Compression eventually occurs at a constant rate, $R_{C}$. Compression and injection may be simultaneous (the RTCMS. . processes) or successive. During pure compression, a vent may be opened at $x=0$, allowing resin to flow out of the mold, or not. Finally, constant or time varying viscosities $\mu$ can be accounted for. All these RTCM processes are analyzed in [13], resulting in 16 models to calculate injection time and maximum mold pressure.

\section{A.2 Calculation of injection time and mold pressure}

The one-dimensional flow of Figure 4 can be analyzed using Darcy's law and a continuity equation which together yield ([19])

$$
\nabla \cdot\left(-\frac{K_{x}}{\mu} \nabla p\right)=-\frac{R_{C}}{H}
$$

where $K_{x}$ is the macroscopic longitudinal permeability (more details are given in Paragraph A.3), $\mu$ is the resin viscosity, $p$ the resin pressure, and $H$ the total plate thickness. If $H, \mu, K_{x}$ and $V_{f}$ depend only on time $t$, Equation (12) can be integrated for $p(x, t)$. The pressure field is quadratic if compression is applied, linear otherwise. The differential equation describing the resin front position also stems from Darcy's law and is

$$
\frac{d x_{F}}{d t}=-\frac{1}{H\left(1-V_{f}\right)}\left(R_{C} x_{F}-\frac{R_{F}}{b}\right),
$$

where $V_{f}$ is the average fiber volume and $b$ is the plate width. A total thickness increment $\Delta H$ is shared among the $N$ plies by looking at the stacking 
sequence as springs of stiffness $E_{2, i}$ in series. If $\Delta H$ is applied to the laminate, the $i$-th ply thickness, $h_{i}$, changes by

$$
\Delta h_{i}=\frac{h_{i} \Delta H}{E_{2, i} \sum_{j=1}^{N} h_{j} / E_{2, j}},
$$

where $E_{2, i}$ is the $i$-th ply transverse Young's modulus. Injection and compression times are calculated by integrating Equation (13). For example, if the injection is pressure controlled at $P$, the front position follows,

$$
H\left(1-V_{f}\right) x_{F}=2 \int_{t_{i}}^{t} \frac{H K_{x} P}{\mu} d s+\left(1-V_{f}(0)\right) H(0) x_{F}\left(t_{i}\right)^{2} .
$$

In the case of the pressure controlled RTM with simultaneous compression (RTCM-SP), the process time $T_{P}$ is solution of the non-linear equation ([13])

$$
a^{2}=\frac{2 P}{\left(R_{C} T_{P}+\left(1-V_{f}(0)\right) H(0)\right)}\left(H(0) I_{1}\left(T_{P}\right)+R_{C} I_{2}\left(T_{P}\right)\right),
$$

where $a$ is the plate length and

$$
I_{1}(t)=\int_{0}^{t} \frac{K_{x}}{\mu} d s \quad, \quad I_{2}(t)=\int_{0}^{t} s \frac{K_{x}}{\mu} d s .
$$

Equation (16) is solved by numerical dichotomy.

\section{A.3 Permeability estimation}

An average plate longitudinal permeability $K_{x}$ is estimated from the plies permeability tensors. Firstly, permeabilities in the principal direction of the $i$-th ply are calculated from the fiber volume fraction using empirical laws

$$
k_{1, i}=A_{1} v_{f, i}^{B_{1}} \quad, \quad k_{2, i}=A_{2} v_{f, i}^{B_{2}} .
$$

Next, the ply longitudinal permeability of the $i$-th ply is obtained by rotating the permeability tensor in the principal direction along the $x$-axis,

$$
k_{x, i}=\frac{k_{1, i}+k_{2, i}}{2}+\frac{k_{1, i}-k_{2, i}}{2} \cos \left(2 \theta_{i}\right),
$$

where $\theta_{i}$ is the $i$-th ply orientation. Finally, ply longitudinal permeabilities are averaged based on the ply thicknesses,

$$
K_{x}=\frac{1}{H} \sum_{i=1}^{N} h_{i} k_{x, i}
$$




\section{B Structure analysis}

\section{B.1 Micromechanical analysis}

Including micromechanical relations in our analysis allows us to study the effect of fiber and matrix properties changes, first on the ply properties, and next on laminate properties through the Classical Lamination Theory outlined in Appendix B.2. Ply mechanical properties are calculated from micromechanical data according to the relations given in [20], where the fibers are assumed to be transversly isotropic. Denoting by $f$ and $m$ fiber and matrix properties, respectively, by $E$ Young's moduli, by $G$ shear stiffness, by $\nu$ Poisson's ratio and by $v_{f}$ the ply fiber volume (a simplification of the $v_{f, i}$ notation of the text for the $i$-th ply), the micro-meso relationships are,

$$
\begin{aligned}
E_{1} & =v_{f} E_{1, f}+\left(1-v_{f}\right) E_{m}, \\
E_{2} & =\frac{E_{m}}{1-\sqrt{v_{f}}\left(1-E_{m} / E_{2, f}\right)}, \\
G_{12} & =\frac{G_{m}}{1-\sqrt{v_{f}}\left(1-G_{m} / G_{12, f}\right)}, \\
\nu_{12} & =v_{f} \nu_{12, f}+\left(1-v_{f}\right) \nu_{m} .
\end{aligned}
$$

\section{B.2 Classical Lamination Theory}

Classical Lamination Theory ([21]) is used to calculate laminate properties, i.e., we apply Kirchhoff plate theory, and neglect through-the-thickness stresses and strains. Under those conditions, the forces $\mathbf{N}$ and moments $\mathbf{M}$

per unit length of the cross section acting on a laminate (cf. Figure 5) are linked to the strains $\varepsilon$ and curvatures $\kappa$ through,

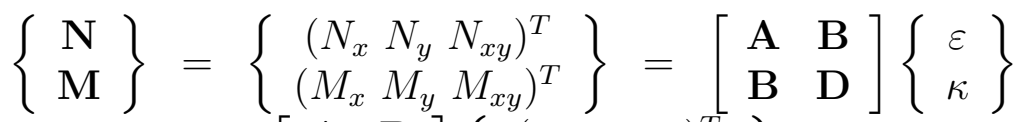

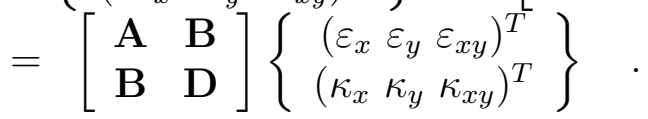

The coefficients of the $3 \times 3$ extensional, coupling and flexural stiffness matrices, A, B, D, respectively, are functions of the material properties, the fiber orientations in each ply, and the position of ply interfaces. The coefficients of the $\mathbf{A}, \mathbf{B}$ and $\mathbf{D}$ matrices can be written as linear combinations of material 
invariants $U_{i}$ 's and lamination parameters $V_{i}$ 's. As the names suggest, the material invariants depend only on the unidirectional material properties $E_{1}$, $E_{2}, G_{12}$ and $\nu_{12}$ of Equations (21-24), while the lamination parameters are only a function of the ply orientations and thicknesses. Detailed expressions of $\mathbf{A}, \mathbf{B}$ and $\mathbf{D}$ using lamination parameters can be found in [22].

The stiffness matrix of Equation (25) can be inverted, which yields the laminate compliance matrix,

$$
\left[\begin{array}{ll}
A & B \\
B & D
\end{array}\right]^{-1}=\left[\begin{array}{cc}
a & b \\
b^{T} & d
\end{array}\right]
$$

The effective Young's moduli of the laminate in the longitudinal, and transverse direction, $E_{x}, E_{y}$, and shear modulus $G_{x y}$ respectively, are expressed as,

$$
E_{x}=\frac{a_{11}}{H} \quad, \quad E_{y}=\frac{a_{22}}{H} \quad, \quad G_{x y}=\frac{a_{66}}{H},
$$

where the index 6 points to the third row/column of the relevant matrix.

\section{B.3 Buckling analysis}

A closed-form solution exists to predict the onset of buckling under the following conditions: the plate is flat, symmetric $(\mathbf{B}=\mathbf{0})$, quasi-orthotropic $\left(D_{16} \approx D_{26} \approx 0\right)$, simply-supported and subject to bi-axial in-plane loads $\left(N_{x}\right.$ and $N_{y}$ only). The plate buckles with $m$ and $n$ half-waves in the $x$ and $y$ directions respectively, when the load reaches $\left(\lambda_{m n} N_{x}, \lambda_{m n} N_{y}\right)$, with

$$
\lambda_{m n}=-\frac{\pi^{2}\left[D_{11}(m / a)^{4}+2\left(D_{12}+2 D_{66}\right)(m / a)^{2}(n / b)^{2}+D_{22}(n / b)^{4}\right]}{(m / a)^{2} N_{x}+(n / b)^{2} N_{y}} .
$$

The critical load factor, $\lambda$, corresponds to the combination of $m$ and $n$ that minimizes $\lambda_{m n}$. If $0<\lambda<1$, the plate fails under the $\left(N_{x}, N_{y}\right)$ loading. 


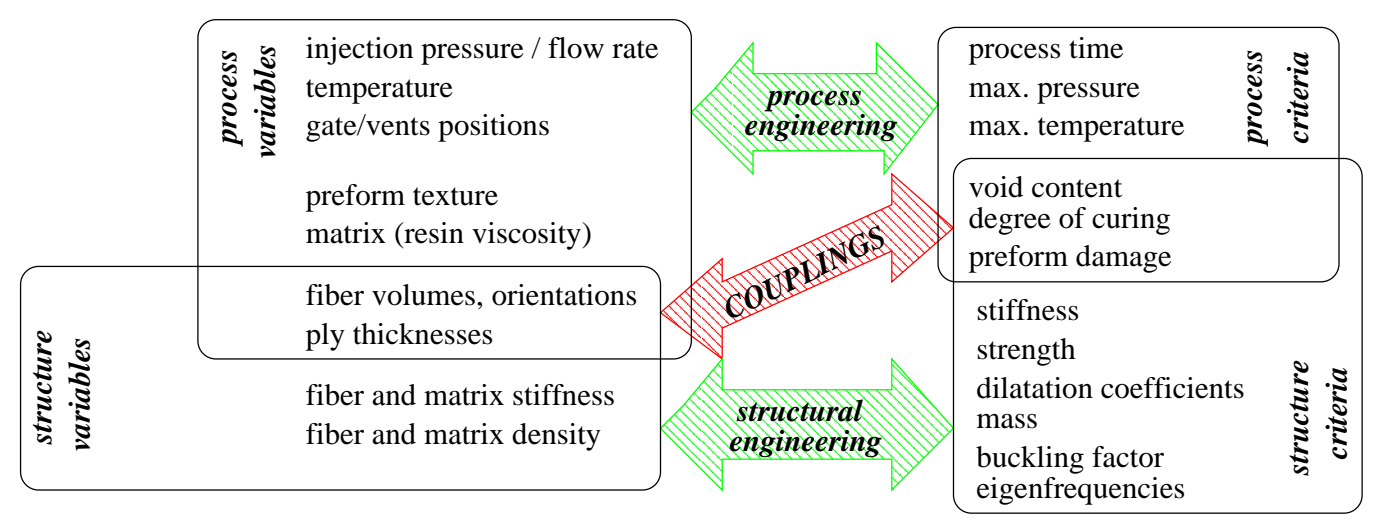

Figure 1: Process and structure couplings. 

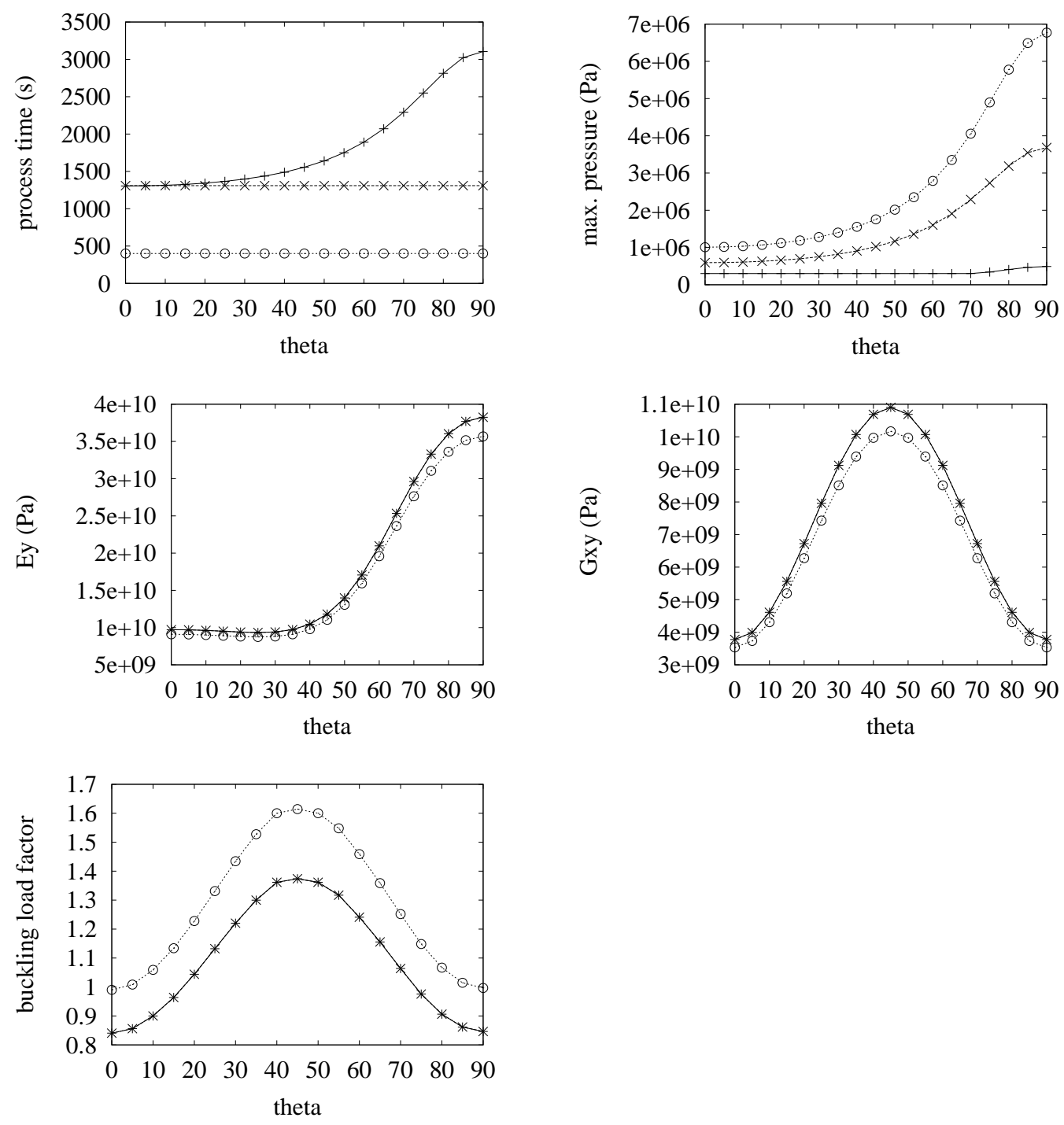

Figure 2: Design criteria vs. $\theta$ for 3 processes, RTCM-P (+), RTCM-F $(\times)$ and RTCM-SF $(\circ),\left(\left( \pm \theta^{\circ}\right)_{2}\right)_{s}, P=3.10^{5} \mathrm{~Pa}, R_{F}=5.510^{-7} \mathrm{~m}^{3} / \mathrm{s}$, $I_{P}=80 \%, R_{C}=1.10^{-6} \mathrm{~m} / \mathrm{s}$. 

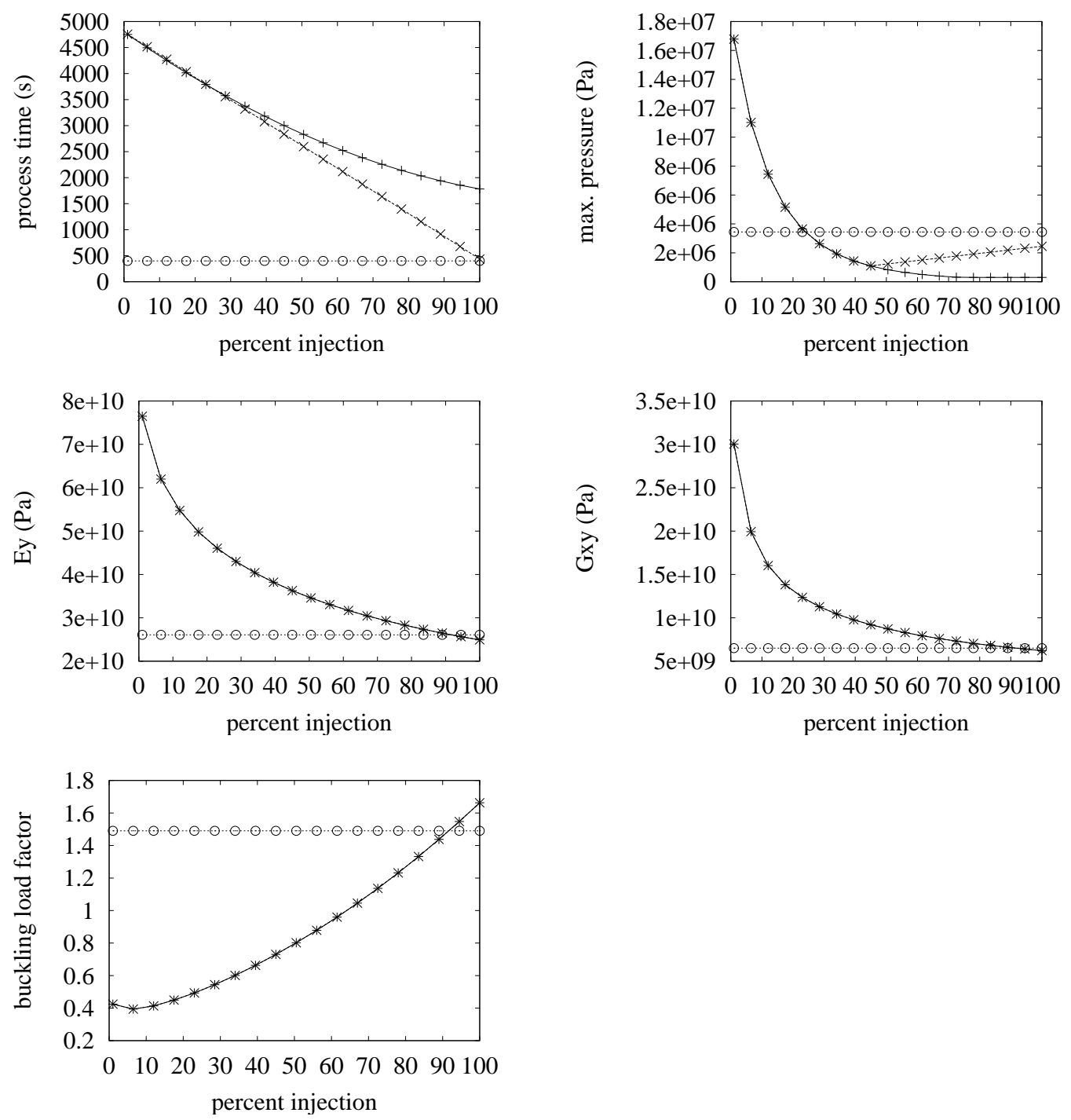

Figure 3: Design criteria vs. percentage of injection length before compression starts for 3 processes, RTCM-P (+), RTCM-F $(\times)$ and RTCM-SF (o), $\left( \pm 54^{\circ} / \pm 90^{\circ}\right)_{s}, P=3.10^{5} \mathrm{~Pa}, R_{F}=5.510^{-7} \mathrm{~m}^{3} / \mathrm{s}, R_{C}=1.10^{-6} \mathrm{~m} / \mathrm{s}$. 


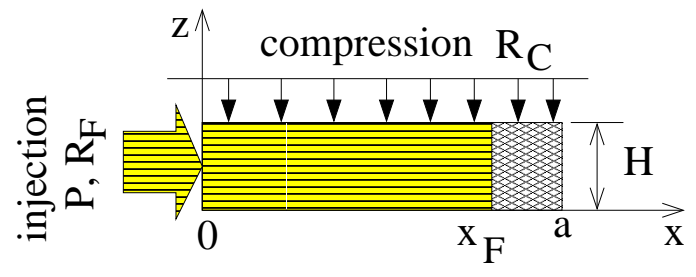

Figure 4: RTCM scenario considered.

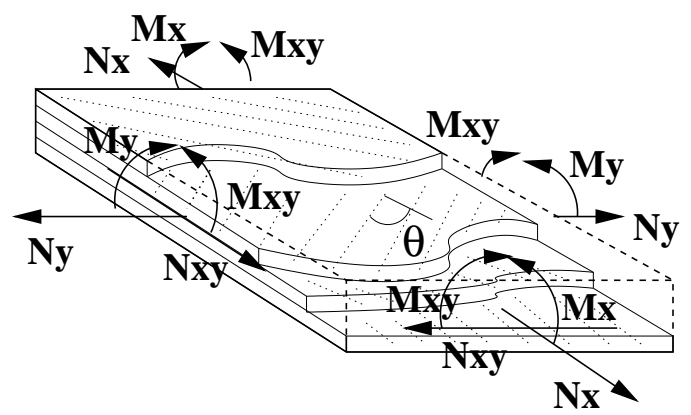

Figure 5: Laminate conventions. 


\begin{tabular}{|c|c|}
\hline \multicolumn{2}{|r|}{ Nomenclature } \\
\hline *, allow & optimal, allowable value \\
\hline$a, b$ & plate length and width \\
\hline$A_{1}, A_{2}, B_{1}, B_{2}$ & permeability law parameters \\
\hline$(C L)$ & coupled low structural performance optimization problem \\
\hline$(C O)$ & coupled optimal structure optimization problem \\
\hline$E_{1, f}, E_{2, f}$ & longitudinal and transverse fiber Young's moduli \\
\hline & resin Young's modulus \\
\hline$E_{y}$ & transverse effective stiffness \\
\hline$G_{12, f}$ & fiber shear stiffness \\
\hline$G_{x y}$ & shear effective stiffness \\
\hline$h_{i}(t)$ & $i$-th ply thickness at time $t$ \\
\hline$H(t)$ & laminate total thickness at time $t$ \\
\hline$I_{P}$ & $\%$ of laminate length filled before compression \\
\hline$K_{x}$ & average longitudinal permeability \\
\hline$\lambda$ & buckling load factor \\
\hline$\mu$ & resin viscosity \\
\hline$\nu_{12, f}, \nu_{m}$ & fiber and resin Poisson's ratio \\
\hline$N_{x}, N_{y}$ & longitudinal, transverse in-plane load \\
\hline$P$ & injection pressure \\
\hline$(P O)$ & process only optimization problem \\
\hline$P_{\max }$ & maximum mold pressure \\
\hline$R_{C}$ & compression rate \\
\hline$R_{F}$ & injection flow rate \\
\hline$(S O)$ & structure only optimization problem \\
\hline$\theta, \theta_{i}$ & fiber orientation (in $i$-th set of plies) \\
\hline$T_{\mathrm{ivn}}$ & inversion time (RTCM-SP process only) \\
\hline$T_{P}$ & total injection time \\
\hline$v_{f, i}(t)$ & $i$-th ply fiber volume fraction at time $t$ \\
\hline$V_{f}(t)$ & average fiber volume fraction at time $t$ \\
\hline$x$ & generic vector of optimization variables \\
\hline
\end{tabular}




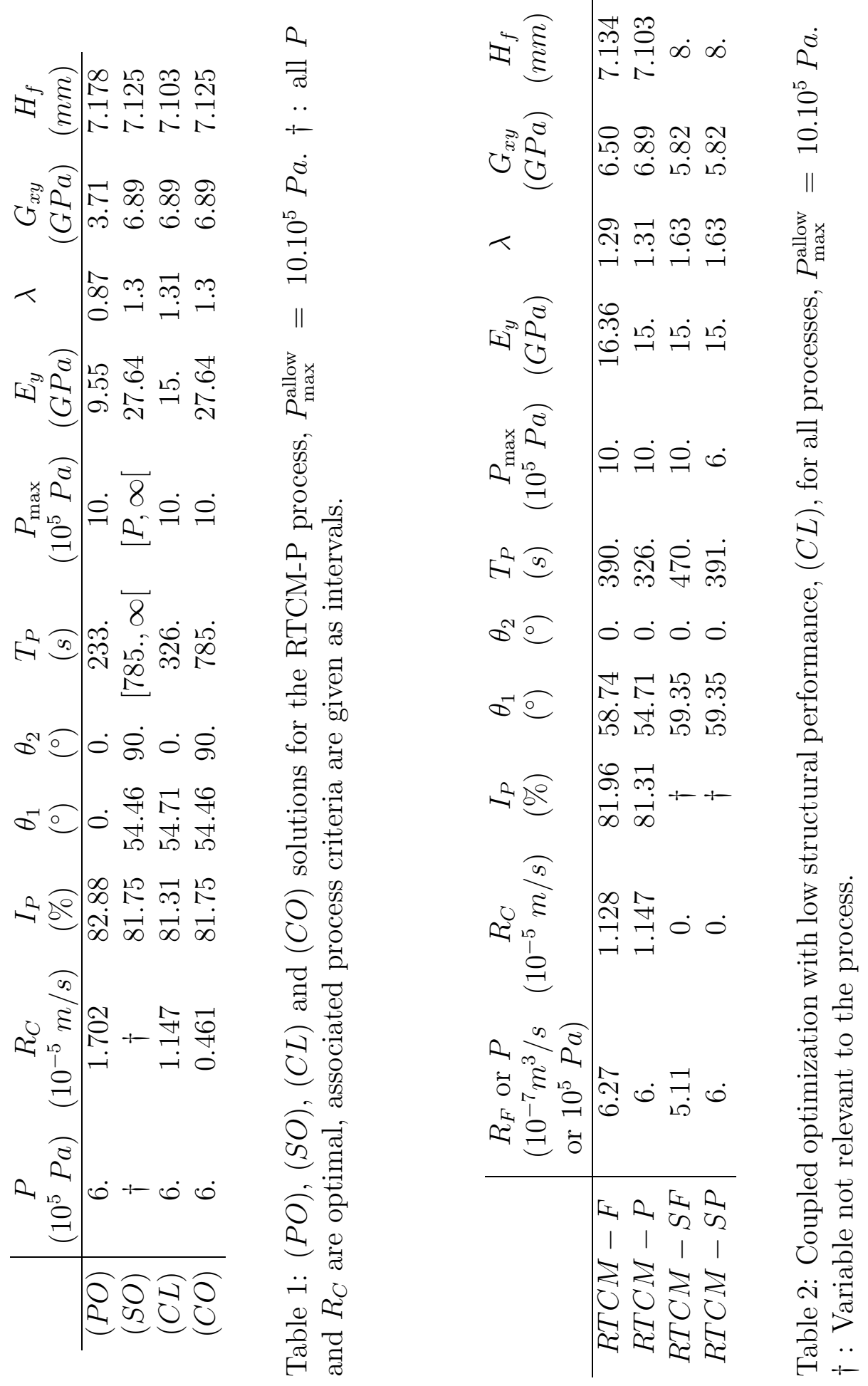




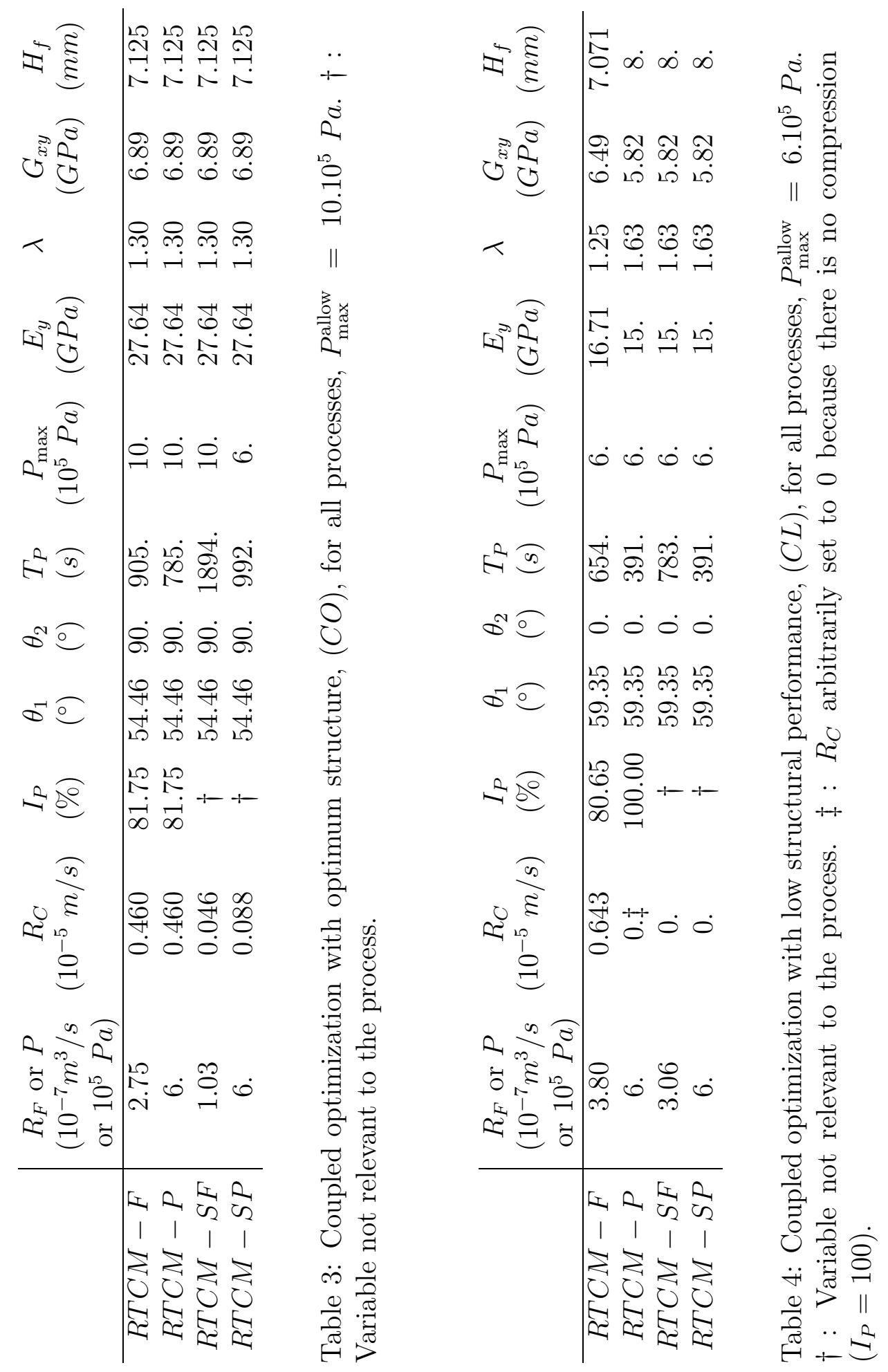




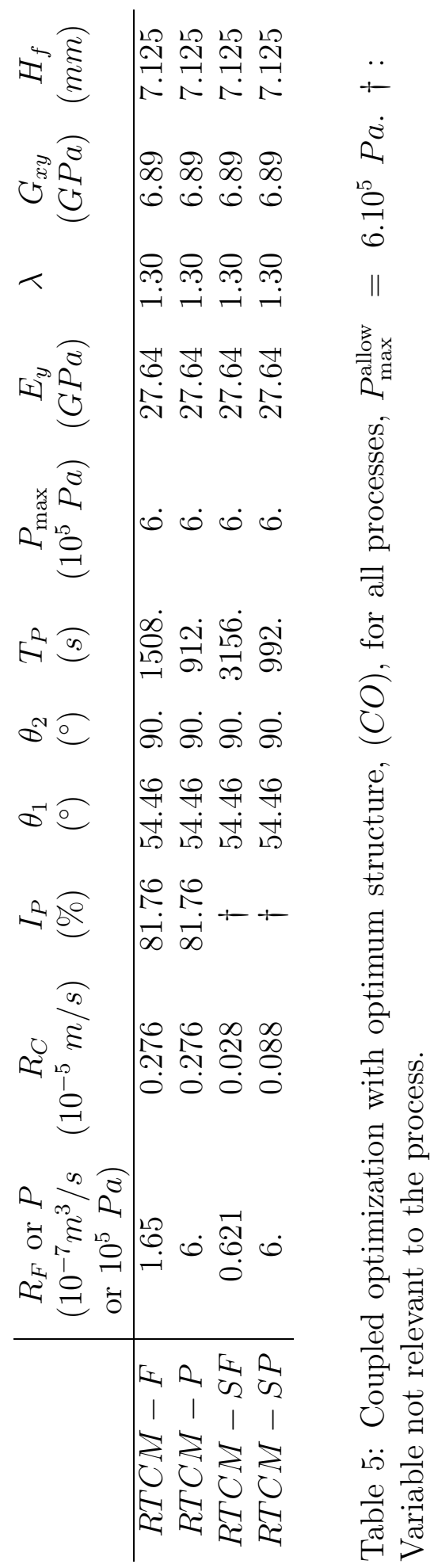

